The Journal of

Thoracic and Cardiovascular

Surgery

Vol 128, No. 4, October 2004

\title{
Reflections on reduction ascending aortoplasty's liveliness
}

Hans H. Sievers, MD

See related article on page 562.
From the Department for Cardiac Surgery, University of Schleswig-Holstein, Campus Luebeck, Department for Cardiac Surgery, Luebeck, Germany.

Received for publication May 26, 2004; accepted for publication June 2, 2004.

Address for reprints: Hans H. Sievers, MD, Chief of Department for Cardiac Surgery, University of Schleswig-Holstein, Campus Luebeck, Department for Cardiac Surgery, Ratzeburger Alle 160, Luebeck, Germany D-23538 (E-mail: herzchir@medinf.muluebeck.de)

J Thorac Cardiovasc Surg 2004;128: $499-501$

$0022-5223 / \$ 30.00$

Copyright (๑) 2004 by The American Association for Thoracic Surgery

doi:10.1016/j.jtcvs.2004.06.014

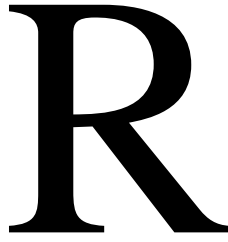

eduction ascending aortoplasty (RAA) is alive, but the real question is whether it should be. Dr Robicsek and colleagues ${ }^{1}$ deserve a lot of credit for renewing the discussion on the appropriate treatment of ascending aortic dilatation-aneurysm. To evaluate RAA's inevitably requires examining the problem of decision making. This is influenced by many factors used to balance the risk and benefit of nonsurgical versus surgical treatment. The risk of the nonsurgical natural course is predominantly determined by the development of aneurysm, as well as by morbidity and mortality caused by dissection and rupture. ${ }^{2}$ The main actors of these events are the wall tension, as calculated by Laplace's law, including pressure, size, and wall thickness, and, furthermore, wall pathology (genetic collagen disorders, chronic dissection, sex, aging, degeneration, and arteriosclerosis), hemodynamic load caused by aortic valve pathology, concomitant aortic valve replacement, and the growth rate of the dilatation. The benefit of the nonsurgical natural course is that those who would never experience the above detrimental events would experience neither its related morbidity and mortality nor the risks of surgical intervention. The risks of surgical intervention are well known, and the benefits are associated with the type of intervention; they are, briefly, total eradication of diseased tissue with a tube graft, reduction of size by RAA, and external reinforcement by wrapping. In addition to all these parameters, life expectancy and individual experience of the surgeon contribute to decision making. Not all of these factors are equally important or easily quantified, but the size of the ascending aorta together with aortic wall morphology are the most important and currently used determinants.

The size of the ascending aorta depends on, among other factors, body surface area (BSA) and, more significantly, age. ${ }^{3}$ There is now general agreement that intervention for ascending aortic aneurysm is indicated in adults at a diameter of greater than $5.5 \mathrm{~cm}$ for degenerative aneurysms. ${ }^{2}$ For patients with Marfan disease and familial ascending aortic aneurysms, the indication is given even for smaller diameters of between 4.5 and $5 \mathrm{~cm}$, especially if size progression is observed. For these large diameters and the special entities, there seems to be no rationale for the RAA because too much diseased tissue is left in place, with an uncertain risk of late complications. A prosthetic tube graft either placed supracommissural or inclusive of the aortic root and, if necessary, extending into the aortic arch is the procedure of choice. The tube graft is relatively simple to sew into place, with 2 circumferential suture lines and relatively low long-term risks, including thromboembolism, false aneurysm at the suture lines, infection, and increased wall stress in the aortic root, possibly promoting the development of aneurysms. ${ }^{4}$ The abovementioned size thresholds, however, are rather crude, without any adjustment for patient conditions. Evolving knowledge on the natural course, especially in patients with Marfan disease, ${ }^{5}$ and a growth rate of $0.07 \mathrm{~cm}$ per year for degenerative dilatation ${ }^{2}$ indicate that there is still a risk for typical complications if smaller dilatations are left untreated. ${ }^{5}$ Together with refined and safer operative techniques and increased 
TABLE 1. Indices for BSA in adults at different ages for determining the size of the ascending aorta in normal

\begin{tabular}{lllll}
\hline BSA $\left(\mathrm{m}^{2}\right)$ Index $\left(\mathrm{cm} / \mathrm{m}^{2}\right)$ & 1.8 & 2.0 & 2.2 & 2.4 \\
$>40 \mathrm{y}$ & 1.9 & 1.8 & 1.7 & 1.6 \\
$<40 \mathrm{y}$ & 1.6 & 1.6 & 1.5 & 1.5
\end{tabular}

$B S A$, Body surface area. The numbers are from Roman et al. ${ }^{3}$

surgical experience, a more aggressive policy for intervention at these lower diameters seems to make sense and is potentially lifesaving provided the risk of operation is low. It must be emphasized, however, that there is neither general consensus nor evidence for this policy. Thus the decision making depends more or less on some single-center reports, unproved hypotheses, surgical experience, and the surgeon's discretion.

To proceed with this policy, we first have to define the normal size that should represent a very low risk for complications. It is useful to define this limit as 2 SDs above the normal average, which presents $95 \%$ of values for normal individuals. Although it would be desirable to integrate the whole diversity of size-determining factors, age and BSA are the most practical and frequently applied. ${ }^{3}$ For the supra-aortic ridge diameter, which closely represents that of the ascending aorta, Roman and coworkers ${ }^{3}$ provided indices for BSA in adults at different ages to calculate the upper normal limit, as shown in Table 1.

It is now possible to take into account the different pathologic entities by defining a special entity factor (eg, 1.10 for Marfan disease, 1.15 for bicuspid aortic valve requiring surgical intervention, 1.20 for concomitant aortic valve replacement, 1.20 for degenerative dilatation with aortic insufficiency, 1.25 for bicuspid valve not requiring valve surgery, and 1.30 for degeneration without valve pathology) to calculate for a given patient the smallest acceptable measured diameter requiring no surgical intervention. For example, in a patient with a bicuspid aortic valve requiring surgical intervention, a BSA of 2.0, and age of 50 years, the calculation for the threshold diameter is as follows: 2.0 (BSA) $\times 1.8$ (Index) $\times 1.15$ (Entity factor $)=$ $4.1 \mathrm{~cm}$. The upper acceptable absolute threshold diameters for this aggressive policy can be defined as $4.0 \mathrm{~cm}$ for Marfan disease, $4.3 \mathrm{~cm}$ for bicuspid valve and for degenerative dilatation with concomitant valve replacement, and 5.0 $\mathrm{cm}$ for degeneration without surgical intervention. Similar values were suggested by Ergin and associates, ${ }^{6}$ who defined the ratio of the measured and mean calculated diameters as threshold values. The major objective of these attractive, liberalized, but aggressive approaches is to tailor the threshold values more closely to the individual's characteristics and operative techniques. Keeping in mind that reconstruction principally preserves physiologic properties, RAA designed to reduce the size to lower than the normal upper diameter limits might have some rationale in these lower size ranges exclusively in patients with poststenotic dilatation, which is characterized by a limited convex extension of dilatation to the right. The physiologic properties potentially being preserved by RAA include the compliance of the ascending aorta aortic impedance and ventricular load, ${ }^{7}$ which is increased in patients with prosthetic tube grafts, ${ }^{8}$ leading to higher cardiac energy costs. ${ }^{9}$ In addition, the most pathologic areas of the dilatation on the right lateral aspect of the wall ${ }^{10}$ can be excluded by RAA most easily performed as a plication between 2 Teflon strips. Tubular dilatations with aortic insufficiency and also dilatations caused by extracellular matrix disorders like Marfan disease do not have a rationale for RAA even not at these lower size thresholds.

It must be considered that many yet unknown factors could affect the outcome of RAA, such as preoperative and postoperative size, cause, surgical technique, experience of the surgeon, redilatation, and reoperation. Some authors report excellent results, but others do not, and the test of time is not proved for RAA. Whether the scope of indication for RAA by wrapping can be extended is rather speculative because the favorable physiologic distensibility of the wrapped aorta is lost, the complexity of the operation is increased, and there is a risk of later dissection possibly triggered by under-the-wrap aortic wall atrophy or wrap displacement. However, it is imaginable that in elderly patients with severe comorbidity, wrapping even without RAA potentially provides some advantages compared with surgical intervention necessitating extracorporeal circulation. When an RAA is performed, it is of particular importance to monitor these patients by means of magnetic resonance imaging every year.

With all due respect, the trilogy of Robicsek's data generation (ie, the authors' own experiences, literature review, and extensive survey) does not provide statistically meaningful data. Only $14.95 \%$ of addressees responded. From a statistical point of view, $85.05 \%$ of contacted surgeons could probably have a different opinion, making the drawn conclusions and statements questionable and without strength for recommendation or generalization. For those surgeons who answered, which is the minority of addressees, RAA is alive but unknown for the rest. Even more importantly, there were no randomized trials. Apart from single-institution experiences, we do not know with certainty whether RAA is a useful operation, which admonishes us to indicate RAA cautiously. But experience is the origin of theory or hypothesis that to verify or falsify is the essence of science. After opening the discussion on different surgical procedures for treatment of ascending aortic dilatation and particularly with respect to a more aggressive approach, it is now time to proceed with the implementation of a working group for a prospective, randomized, and monitor-controlled trial and a reliable registry to generate statistically valid data. 
For that, we need fruitful collaboration under the auspices of our societies to generate evidence-based guidelines for the treatment of ascending aortic dilatation-aneurysm to optimize the care of our patients.

\section{References}

1. Robicsek F, Cook JW, Reames MK, Sr, Skipper ER. Size reduction ascending aortoplasty: is it dead or alive? J Thorac Cardiovasc Surg. 2004;128:562-70.

2. Elefteriades JA. Natural history of thoracic aortic aneurysms: indications for surgery, and surgical versus nonsurgical risks. Ann Thorac Surg. 2002;74(suppl):S1877-80.

3. Roman MJ, Devereux RB, Kramer-Fox R, O'Loughlin J. Twodimensional echocardiographic aortic root dimensions in normal children and adults. Am J Cardiol. 1989;64:507-12.

4. Simon-Kupilik N, Schima H, Huber L, Moidl R, Wipplinger G, Losert $\mathrm{U}$, et al. Prosthetic replacement of the aorta is a risk factor for aortic root aneurysm development. Ann Thorac Surg. 2002;73:455-9.

5. Leggert ME, Unger TA, O'Sullivan CK, Zwink TR, Bennett RL, Byers PH, et al. Aortic root complications in Marfan's syndrome: identification of a lower risk group. Heart. 1996;75:389-95.

6. Ergin MA, Spielvogel D, Apaydin A, Lansman SL, McCullough JN, Galla JD, et al. Surgical Treatment of the dilated ascending aorta: when and how? Ann Thorac Surg. 1999;67:1834-9.

7. Milnor WR. Arterial impedance as ventricular afterload. Circ Res. 1975;36:565-70.

8. Bauernschmitt R, Schulz S, Schwarzhaupt A, Kiencke U, Vahl CF, Lange R, et al. Simulation of arterial hemodynamics after partial prosthetic replacement of the aorta. Ann Thorac Surg. 1999;67:67682.

9. Kim SY, Hinkamp TJ, Jacobs WR, Lichtenberg RC, Posniak H, Pifarre R. Effect of an inelastic aortic synthetic vascular graft on exercise hemodynamics. Ann Thorac Surg. 1995;59:981-9.

10. Cotrufo M, De Santo LS, Esposito S, Renzulli A, Della Corte A, De Feo M, et al. Asymmetric medial degeneration of the intrapericardial aorta in aortic valve disease. Int J Cardiol. 2001;81:37-41.

To assure fairness to authors submitting work for consideration in The Journal of Thoracic and Cardiovascular Surgery, a mechanism exists for managing conflicts of interest. The editor and each of the section editors complete a "Conflict of Interest" form that identifies any and all relationships with commercial and other academic entities. When the editor has a potential conflict because of a relationship with another entity or author, the editor appoints an alternate editor from among the section editors or editorial board members who assumes the entire responsibility for final decisions on the manuscript in question. The editor does not read the reviews that are submitted nor engage in discussing the manuscript prior to the final decision. When the conflict of interest involves a section editor, a "guest section editor" is appointed who fills the role normally played by the conflicted section editor. All members of the editorial board and reviewers are asked to indicate any conflict of interest when they agree to review a manuscript. 